\title{
ON THE FORMAL GROUPS APPROACH TO COBORDISM
}

\author{
SANDRO BUONCRISTIANO ${ }^{1}$ AND DEREK HACON ${ }^{2}$
}

\begin{abstract}
We give a direct proof of the fact that the complex bordism ring $U_{*}$ is a polynomial ring, based on Quillen's approach. The proof is self-contained, apart from the assumption that the groups $U_{n}$ are all finitely generated and an algebraic lemma on formal groups.
\end{abstract}

1. Preliminaries. $U$-structures. A $U$-structure on a map $f: X \rightarrow Y$ between (paracompact, smooth) manifolds is a homotopy class of complex structures on $\nu_{X} \oplus f^{*} \tau_{Y}$ where $\nu_{X}$ is the normal bundle of $X$ in $\mathbf{R}^{N}$ (suitable $N$ ) and $\tau_{Y}$ is the tangent bundle of $Y$. When $Y=p t$ we have a $U$-structure on $X$, which may also be specified by a complex structure on $\tau_{X} \oplus \varepsilon$ ( $\varepsilon$ a real trivial bundle of suitable dimension). A $U$-manifold is a manifold with $U$-structure and a $U$-map is a map with $U$-structure. Clearly,

(i) the composite of two $U$-maps is a $U$-map,

(ii) any map between $U$-manifolds is a $U$-map, in the obvious way,

(iii) any map homotopic to a $U$-map is a $U$-map.

Bordism and cobordism. For a pair of spaces $X, A$, bordism $\Omega_{*}(X, A)=$ $\oplus \Omega_{n}(X, A)$ is defined as usual by compact $U$-manifolds modulo $U$-bordism. If $f$ : $X, A \rightarrow Y, B$ is a map we define $f_{*}$ by $f_{*}[g]=[f \circ g]$ where $g: M, \partial M \rightarrow X, A$.

Let $X$ be a manifold. Define $U_{n}(X)$ by $U$-maps $M \rightarrow X$ (where $\operatorname{dim} M=n$ and $M$ is closed (= compact, boundaryless)) modulo $U$-bordism of $U$-maps. Thus, for $X$ closed, $U_{*}(X)$ is the usual complex cobordism (but graded by dimension) and, for $X$ a $U$-manifold, $U_{*}(X)=\Omega_{*}(X)$, in the obvious way. If $f: X \rightarrow Y$ is a $U$-map then $f_{*}: U_{*}(X) \rightarrow U_{*}(Y)$ may be defined by $f_{*}[g]=[f \circ g]$. If $h: X \rightarrow Y$ is a proper smooth map, define $h^{*}: U_{*}(Y) \rightarrow U_{*}(X)$ as follows. If $M$ is closed and $g: M \rightarrow Y$ a $U$-map we may assume (modulo homotopy) that $g$ is transverse to $h$ and consider the pull-back diagram

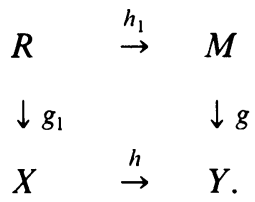

$R$ is closed and $g_{1}$ is a $U$-map. Define $h^{*}[g]=\left[g_{1}\right]$ (independent of choice of $g$ ).

Received by the editors January 25, 1985 and, in revised form, June 8, 1985.

1980 Mathematics Subject Classification. Primary 55N22, 57R77; Secondary 57R20, 57R22, 57R85.

$\mathrm{Key}$ words and phrases. Complex cobordism, Euler class, $\mathrm{Z} / \mathrm{p}$-action, equivariant complex structure, formal group law.

${ }^{1}$ Research supported by M.P.I.

2 Research supported by F.I.N.E.P. 
Lemma 1. (i) For U-maps, $(f \circ g)_{*}=f_{*} \circ g_{*}$.

(ii) For proper maps, $(h \circ k)^{*}=k^{*} \circ h^{*}$.

(iii) $f_{*}$ is invariant under homotopy.

(iv) If $h: X \rightarrow Y$ is proper and $g: W \rightarrow Y$ is a $U$-map with $h, g$ transverse, then, in the pull-back diagram,

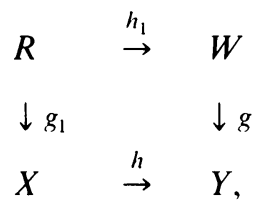

$h_{1}$ is proper, $g_{1}$ is a U-map and $h^{*} \circ g_{*}=g_{1 *} \circ h_{1}^{*}$.

We write $U_{*}=U_{*}(p t)=\Omega_{*}(p t)$. $U_{*}(X)$ is a $U_{*}$-algebra (with unit $1_{X}$ if $X$ is closed). If $f: X \rightarrow Y$ is proper then $f^{*}$ is a $U_{*}$-algebra map (with $f^{*} 1_{Y}=1_{X}$ if $X$, $Y$ closed).

If $E$ is a complex bundle over a closed manifold $X$ then the zero-section $i$ : $X \rightarrow E$ is a $U$-map. The Euler class $e(E)$ is $i^{*} i_{*} 1_{X}$ in $U_{*}(X)$. We have $e\left(E_{1} \oplus E_{2}\right)$ $=e\left(E_{1}\right) \cdot e\left(E_{2}\right)$ and $e\left(f^{*} E\right)=f^{*} e(E)$.

Let $\zeta=S^{2 m+1} \times{ }_{S^{1}} \mathbf{C}$ be the dual of the canonical line-bundle over $\mathbf{C P}(m)$ and let $z=e(\zeta)$. An easy argument, involving the Thom isomorphism, shows that

$$
U_{*}(\mathbf{C P}(m)) \simeq U_{*}[t] / t^{m+1}=0
$$

(isomorphism of $U_{*}$-algebras taking $t$ to $z$ ).

Lens-spaces. Let $p \geqslant 2$ and $L=S^{2 m+1} / \Gamma$ where $\Gamma(v)=\exp (2 \pi i / p) v$. If $X$ is a manifold with smooth $\mathbf{Z} / p$-action $T$, write $L \stackrel{\sim}{\times} X$ for $S^{2 m+1} \times{ }_{\mathbf{z} / p} X$, where the action on $S^{2 m+1} \times X$ is $\Gamma \times T$. $L \times X$ fibers over $L$ with fiber $X$.

Suppose $X \rightarrow Y$ is an equivariant embedding ( $X, Y$ manifolds with smooth $\mathbf{Z} / p$-actions) whose normal bundle has an equivariant complex structure. Then the induced embedding $L \underset{\sim}{\times} X \rightarrow L \stackrel{\sim}{\times} Y$ has a complex structure on its normal bundle and is thus a $U$-map.

$L$ is a $U$-manifold via the equivariant complex structure on $\tau_{S^{2 m+1}} \oplus \mathbf{R}$ coming from $\mathbf{C}^{m+1}$. If $\boldsymbol{\theta}$ is a complex linear action of $\mathbf{Z} / p$ (on a vector space $V$, say) we write $L \times \underset{\sim}{\theta}$ for the complex bundle $L \times \underset{\sim}{ }$. For example, if $\omega$ acts on $\mathbf{C}$ by $\omega(z)=\exp (2 \pi i / p) z$, then $L \underset{\sim}{\times} \omega$ is the dual of the canonical line bundle. Clearly, $L \times\left(\omega^{j}\right) \simeq(L \times \underset{\sim}{\times} \omega)^{j} \quad(j$-fold $\otimes)$. Let $\rho$ be the action $\rho\left(z_{1}, z_{2}, \ldots, z_{p}\right)=$ $\left(z_{2}, \ldots, z_{p}, z_{1}\right)$ on $\mathbf{C}^{p}$. Then $\rho=$ diagonal $\oplus \nabla$ where $\nabla \simeq \omega \oplus \omega^{2} \oplus \cdots \oplus \omega^{p-1}$, and $L \stackrel{\sim}{\times} \nabla \simeq q^{*} \Sigma$ where $\Sigma=\zeta \oplus \zeta^{2} \oplus \cdots \oplus \zeta^{p-1}$ and $q: L \rightarrow \mathbf{C P}(m)$ is the quotient map. We may identify $q$ with the projection of the circle-bundle associated to $\zeta^{p}$. Then the following piece of the Gysin sequence

$$
\cdots \rightarrow U_{*}(\mathbf{C P}(m)) \stackrel{e\left(\zeta^{p}\right)}{\rightarrow} U_{*}(\mathbf{C P}(m)) \stackrel{q^{*}}{\rightarrow} U_{*}(L) \rightarrow \cdots
$$

is exact at the middle term.

Splitting principle. Let $T(n)=$ diagonal matrices in $U(n)$ and $B T(n) \rightarrow B U(n)$ be induced by $T(n) \subseteq U(n)$. 
LEMMA 2. $\Omega_{*}(B T(n)) \rightarrow \Omega_{*}(B U(n))$ is onto.

Proof. The proof follows from the following well-known facts.

(a) $B U(n)$ has a C.W. decomposition in which every cell is a cycle,

(b) $H_{*}(B T(n) ; \mathbf{Z}) \rightarrow H_{*}(B U(n) ; \mathbf{Z})$ is onto,

(c) $\Omega_{*}(B T(n)) \rightarrow H_{*}(B T(n) ; \mathbf{Z})$ is onto,

together with an induction up the skeletons (taking $B T(n) \rightarrow B U(n)$ to be cellular).

Now a $\mathbf{C}^{n}$-bundle $E$ over a closed $U$-manifold defines $[E] \in \Omega_{*}(B T(n))$. From Lemma 2 we deduce that (splitting principle) every element of $\Omega_{*}(B U(n))$ may be written as $[E]$ where $E$ is a $\oplus$ of complex line bundles.

Formal group law. There exist $a_{i j} \in U_{*}$ (for all $i, j>0$ ) such that, for any two complex line bundles $L_{1}, L_{2}$ over a closed manifold,

$$
e\left(L_{1} \otimes L_{2}\right)=e\left(L_{1}\right)+e\left(L_{2}\right)+\sum a_{i j} e\left(L_{1}\right)^{i} e\left(L_{2}\right)^{j}
$$

If, in $U_{*}(X)$, we write $u \sim v$ to mean that $u-v \in \sum a_{i j} U_{*}(X)$ (i.e., $u-v$ is a sum of elements each of which is divisible by at least one $a_{i j}$ ), then we have

$$
e\left(L_{1} \otimes L_{2}\right) \sim e\left(L_{1}\right)+e\left(L_{2}\right) \text {. }
$$

The main result we will prove (in $\S 2$ ) is

THEOREM (QUILLEN). $1 \in U_{0}$ and the $a_{i j}$ generate $U_{*}$ as a ring.

Characteristic numbers. To get relations in $U_{*}$ we will use the splitting principle together with certain cobordism characteristic numbers.

Define $\Phi: \Omega_{n}(B U(n)) \rightarrow U_{n}(\mathbf{C P}((p-1) n)$ ) (a close relative of the Steenrod-tom Dieck $p$ th power operation) by the formula $\Phi(E)=\pi_{*} e(\Sigma \otimes E)$ where $E$ is a bundle over $M, \pi: P \times M \rightarrow P$ is projection, $P=\mathbf{C} P((p-1) n), \otimes$ is external $\otimes$, $\Sigma=\zeta \oplus \cdots \oplus \zeta^{p-1}$ and $p \geqslant 2$.

LEMMA 3. $\Phi$ is well defined (i.e., bordism invariant).

Proof. Let $B$ be a $U$-bordism between $M$ and $N$ (say), $i: M \rightarrow B, j: N \rightarrow B$ inclusions and $E$ a bundle over $B$. Then, in $U_{*}(P \times B)$, we have

$$
(1 \times i)_{*} e\left(\Sigma \otimes i^{*} E\right)=(1 \times j)_{*} e\left(\Sigma \otimes j^{*} E\right),
$$

by relative transversality. Applying $\pi_{*}$ we get the lemma.

DEFINITION. Define $\phi: \Omega_{n} \rightarrow U_{n}\left(=\Omega_{n}\right)$ by $\phi(M)=$ coefficient of $z^{(p-1) n}$ in $\Phi\left(\nu_{M}\right)$ where $\nu_{M}$ is the complex normal bundle of the $n$-dimensional $U$-manifold $M$ in (for convenience) $\mathbf{R}^{3 n}$. By Lemma $3, \phi$ is well defined (since $[M]=[N]$ implies $\left.\left[\nu_{M}\right]=\left[\nu_{N}\right]\right)$.

LEMMA 4. $\phi(M) \sim s[M]$ where $s=(p-1) !^{n}$.

Proof. By the splitting principle we have $\left[\nu_{M}\right]=\left[L_{1} \oplus \cdots \oplus L_{n}\right]$ in $\Omega_{n}(B U(n))$ where $L_{1}, \ldots, L_{n}$ are complex line bundles over $N$, say. Of course, $[N]=[M]$. 
Writing $\times$ for external product in $U_{*}(P \times N)$ we have

$$
\begin{aligned}
e\left(\Sigma \otimes\left(L_{1} \oplus \cdots \oplus L_{n}\right)\right) & \sim \prod_{k, l}\left(k z \times 1_{N}+1_{P} \times e\left(L_{l}\right)\right) \\
& =s\left(z^{(p-1) n} \times 1_{N}\right)+\text { lower powers of } z .
\end{aligned}
$$

Applying $\pi_{*}$ and taking coefficients of $z^{(p-1) n}$ gives the lemma.

(Note $s=(p-1) !^{n}$ since $1 \leqslant k \leqslant p-1$ and $1 \leqslant l \leqslant n$.)

Proposition 1. If $n>0$ then $\phi(M) \sim$ an element of $p U_{n}$.

Proof. Since $U_{1}=0$ we may take $n \geqslant 2$. Write $V=\mathbf{R}^{3 n}$ and take the normal bundle $E$ of $M$ to be embedded in $V$. Thus $M \subset E \subset V$. In the pullback diagram

$$
\begin{array}{cll}
L \times M & \stackrel{\pi}{\rightarrow} & L \\
\downarrow q \times 1 & & \downarrow q \\
P \times M & \stackrel{\pi}{\rightarrow} & P
\end{array}
$$

we have $q^{*} \circ \pi_{*}=\pi_{*} \circ(q \times 1)^{*}$, by Lemma 1 . Thus

$$
\begin{aligned}
q^{*} \Phi(E) & =q^{*} \pi_{*} e(\Sigma \otimes E)=\pi_{*}(q \times 1)^{*} e(\Sigma \otimes E) \\
& =\pi_{*} e\left(q^{*} \Sigma \otimes E\right)=\pi_{*} e((L \underset{\sim}{\times} \nabla) \otimes E) .
\end{aligned}
$$

We claim that $q^{*} \Phi(E)=0$. Granted that $q^{*} \Phi(E)=0$, it follows, from the Gysin sequence for $\zeta^{p}$, that $\Phi(E)$ is a multiple of $e\left(\zeta^{p}\right)$ in $U_{*}(P)$ and hence that $\Phi(E) \sim$ an element of $p U_{*}(P)$. Comparing coefficients of $z^{(p-1) n}$, we deduce that $\phi(M) \sim$ an element of $p U_{*}$, which proves the proposition.

To show that $q^{*} \Phi(E)=0$ it suffices to show that $j_{*} e((L \underset{\sim}{\times} \nabla) \otimes E)=0$, where $j: L \times M \rightarrow L \times V$ (and $J: L \underset{\sim}{\times} M^{p} \rightarrow L \underset{\sim}{\times} V^{p}$ below) are embeddings induced by $M \subset V$. We may identify $(L \times \nabla) \otimes E$ with $L \times(\nabla \otimes E)$ since both are quotients of $\left(S \times \mathbf{C}^{p-1}\right) \otimes E=S \times\left(\mathbf{C}^{p-1} \otimes E\right)$ by the same relation. We get the commutative diagram

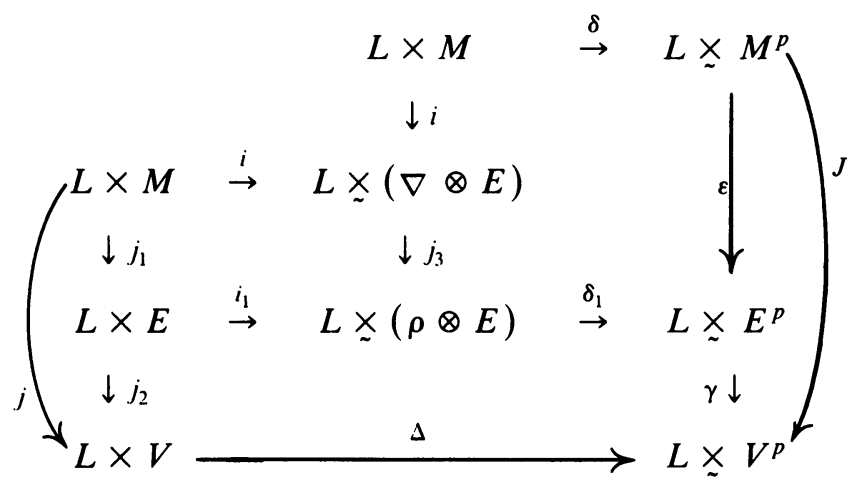

in which $M^{p}$ etc. stand for $M \times \cdots \times M$ etc. with actions $\left(m_{1}, m_{2}, \ldots, m_{p}\right) \rightarrow$ $\left(m_{2}, \ldots, m_{p}, m_{1}\right)$ etc. and all maps are induced by the obvious inclusions, zero-sections or diagonal maps. 
Clearly all horizontal maps are proper and all vertical maps are $U$-maps.

Let us consider the three rectangles:

(1)

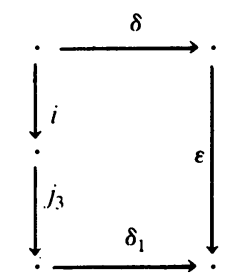

(2)

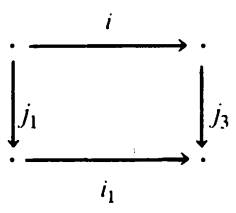

(3)

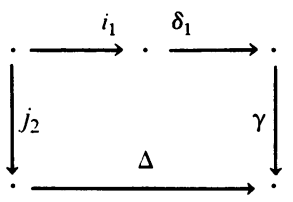

We claim that the above rectangles are pull-back diagrams. As for (1) consider $L \times E^{p}$. This is a bundle over $L \underset{\sim}{\times} M^{p}$. Its restriction to the diagonal $L \times M$ is $L \underset{\sim}{\tilde{x}}(E \oplus \cdots \oplus E)$, i.e. $L \underset{\sim}{\times}(\rho \otimes \tilde{E})$. Clearly, in $L \underset{\sim}{\times} E^{p}$ the zero section $L \underset{\sim}{\times} M^{p}$ and (the total space of) the bundle $L \times(\rho \otimes E)$ intersect transversally in the zero section of the bundle $L \underset{\sim}{\times}(\rho \otimes E)$, which is $L \times M$. This proves that (1) is a pull-back diagram. As for (2), it is enough to observe that the bundle $L \times(\rho \otimes E)$ over $L \times M$ splits into the Whitney sum of the "diagonal" bundle $L \times E$ and the "antidiagonal" bundle $L \underset{\sim}{\times}(\nabla \otimes E)$. Finally, (3) is clearly a pull-back diagram. Applying Lemma 1 repeatedly we have the identity

$$
j_{*} i * i_{*} 1_{L \times M}=\Delta^{*} J_{*} 1_{L \times M^{p}} .
$$

In fact,

$$
\begin{aligned}
j_{*} i^{*} i_{*} 1_{L \times M} & =j_{*} i^{*} i_{*} \delta^{*} 1_{L \times M^{p}} \\
& =j_{2 *}\left(j_{1 *} i^{*}\right) i_{*} \delta^{*} 1_{L \times M^{p}} \\
& =j_{2 *}\left(i_{1}^{*} j_{3 *}\right) i_{*} \delta^{*} 1_{L \times M^{p}} \quad \text { (by Lemma 1) } \\
& =j_{2 *} i_{1}^{*}\left(j_{3} i\right)_{*} \delta^{*} 1_{L \times M^{p}} \\
& =j_{2 *} i_{1}^{*} \delta_{1}^{*} \varepsilon_{*} 1_{L \subseteq M^{p}} \quad(\text { by Lemma } 1) \\
& =j_{2 *}\left(\delta_{1} i_{1}\right)^{*} \varepsilon_{*} 1_{L \times M^{p}} \\
& =\Delta^{*} \gamma_{*} \varepsilon_{*} 1_{L \times M^{p}} \quad(\text { by Lemma } 1) \\
& =\Delta^{*}(\gamma \varepsilon)_{*} 1_{L \times M^{p}}=\Delta^{*} J_{*} 1_{L \times M^{p}}
\end{aligned}
$$

Now $J$ is homotopic to the composite of projection $L \underset{\sim}{\times} M^{p} \rightarrow L$ and inclusion $L \rightarrow L \stackrel{\sim}{\times} V^{p}$. But $\operatorname{dim}\left(L \times V^{p}\right)-\operatorname{dim} L-\operatorname{dim}(L \times V)=(p-1) n-1$ is $>0$ (since we took $p, n \geqslant 2$ ). Thus $\Delta^{*} J_{*}=0$ and the proposition is proved.

2. Proof of the theorem. Let $n>0$ and $p \geqslant 2$ be prime. Then $\phi(M) \sim$ an element of $p U_{n}$ (Proposition 1) and $\phi(M) \sim s[M]$, where $s=(p-1) !^{n}$ (Lemma 4). Therefore, $[M] \sim$ an element of $p U_{n}$. 
Let $C_{*}$ be the ideal $\sum a_{i j} U_{*}$ of $U_{*}$. For all $n \geqslant 0, U_{n} / C_{n}$ is finitely generated since $U_{n}$ is. We showed above that for $n>0$ any prime $p \geqslant 2$ divides any element of $U_{n} / C_{n}$. Thus, $U_{n} / C_{n}=0$ for $n>0$.

Since no $a_{i j}$ lies in $U_{0}=\mathbf{Z}$ we may repeatedly substitute $U_{0}+C_{*}$ for $U_{*}$ in the definition $C_{*}=\sum a_{i j} U_{*}$ to deduce that the $a_{i j}$ generate $\oplus_{n>0} U_{n}$ as a ring. This proves the theorem.

3. Polynomial structure of $U_{*}$. In $\S 2$ we saw that $U_{*}=$ subring $L$ generated by $1 \in U_{0}$ and the $a_{i j}$. A calculation involving the defining properties of formal groups [K] shows that there is a (ring) epimorphism $\mathbf{Z}\left[t_{2}, t_{4}, t_{6}, \ldots\right] \rightarrow L$ (taking $t_{2 i}$ into $U_{2 i}$ ) and hence an epimorphism $\mathbf{Z}\left[t_{2}, t_{4}, t_{6}, \ldots\right] \rightarrow U_{*}$. But, by a well-known Chernnumbers calculation [MS], the rank of $U_{2 r}$ is at least the number of partitions of $r$. Thus we have a ring isomorphism $\mathbf{Z}\left[t_{2}, t_{4}, t_{6}, \ldots\right] \simeq U_{*}$.

The unoriented analogue of Proposition 1 is proved similarly. The logarithm for unoriented bordism $N_{*}$ then provides an epimorphism $(\mathbf{Z} / 2)\left[t_{2}, t_{4}, t_{5}, t_{6}, \ldots\right] \rightarrow N_{*}$ (where $\left.t_{i} \rightarrow N_{i}(i \neq 1,3,7, \ldots)\right)$ and a Stiefel-Whitney numbers calculation [MS] shows this to be an isomorphism.

\section{REFERENCES}

[K] M. Karoubi, Cobordisme et groupes formels (d'après D. Quillen et T. tom Dieck), Sém. Bourbaki, Lecture Notes in Math., vol. 472, Springer-Verlag, Berlin and New York, 1973.

[MS] J. Milnor and J. Stasheff, Lectures on characteristic classes, Ann. of Math. Stud., vol. 76, Princeton Univ. Press, Princeton, N. J., 1974.

[Q] D. Quillen, Elementary proofs of some results of cobordism theory using Steenrod operations, Adv. in Math. 7 (1971), 29-56.

Dipartimento di Matematica, Seconda Universita di Roma, Roma, Italy

P.U.C. do Rio de Janeiro, RuQ Marques de Sao Vicente 225, CEP 22453, Rio de Janeiro, Brazil (Current address of Derek Hacon)

Current address (Sandro Buoncristiano): Dipartimento di Matematica, Università di Tor Vergata, Via Orazio Raimondo, 00173 Roma, Italy 